\title{
Direct Profiling of Phytochemicals in Tulip Tissues and In Vivo Monitoring of the Change of Carbohydrate Content in Tulip Bulbs by Probe Electrospray Ionization Mass Spectrometry
}

\author{
Zhan Yu, ${ }^{\text {a,e }}$ Lee Chuin Chen, ${ }^{\text {a }}$ Hiroaki Suzuki, ${ }^{\text {a,b }}$ Osamu Ariyada, ${ }^{\mathrm{b}}$ \\ Rosa Erra-Balsells, ${ }^{c}$ Hiroshi Nonami, ${ }^{d}$ and Kenzo Hiraoka ${ }^{a}$ \\ ${ }^{a}$ Clean Energy Research Center, University of Yamanashi, Kofu, Japan \\ b ARIOS Inc., Akishima, Tokyo, Japan \\ ${ }^{\mathrm{c}}$ CIHIDECAR-CONICET, Departamento de Quimica Organica, Facultad de Ciencias Exactas y Naturales, \\ Universidad de Buenos Aires, Buenos Aires, Argentina \\ d Plant Biophysics/Biochemistry Research Laboratory, Faculty of Agriculture, Ehime University, Matsuyama, \\ Japan \\ e College of Chemistry and Biology, Shenyang Normal University, Shenyang, China
}

Probe electrospray ionization (PESI) is a recently developed ESI-based ionization technique which generates electrospray from the tip of a solid needle. In this study, we have applied PESI interfaced with a time of flight mass spectrometer (TOF-MS) for direct profiling of phytochemicals in a section of a tulip bulb in different regions, including basal plate, outer and inner rims of scale, flower bud and foliage leaves. Different parts of tulip petals and leaves have also been investigated. Carbohydrates, amino acids and other phytochemicals were detected. A series of in vivo PESI-MS experiments were carried out on the second outermost scales of four living tulip bulbs to monitoring the change of carbohydrate content during the first week of initial growth. The breakdown of carbohydrates was observed which was in accordance with previous reports achieved by other techniques. This study has indicated that PESI-MS can be used for rapid and direct analysis of phytochemicals in living biological systems with advantages of low sample consumption and little sample preparation. Therefore, PESI-MS can be a new choice for direct analysis/profiling of bioactive compounds or monitoring metabolic changes in living biological systems. (J Am Soc Mass Spectrom 2009, 20, 2304-2311) (C) 2009 Published by Elsevier Inc. on behalf of American Society for Mass Spectrometry

$\mathrm{T}$ The demand of new drug research speeds the study of natural plant products because nearly $25-50 \%$ of current pharmaceuticals in use are derived from natural plant products [1]. As plants have served as a major source of drugs, many kinds of analytical methods have been applied to discover new candidate compounds which can be directly used or structurally modified as new drugs. Conventional analysis of natural plant products is a time-consuming and labor-extensive process. General steps include extraction by using water or organic solvents, separation by column chromatography or solidphase extraction, and structural characterization by using instrumental methods. The bioactive constituents, especially some trace amounts of compounds, are often damaged or lost in the long and complicated preparation process. Thus, it is necessary to develop direct analysis methods which can be used to characterize and analyze phytochemicals in plants directly. Specially, direct analy-

Address reprint requests to Professor K. Hiraoka, Clean Energy Research Center, University of Yamanashi, 4-3-11 Takeda, Kofu, Yamanashi 400-8511, Japan. E-mail: hiraoka@yamanashi.ac.jp sis methods will be capable of giving direct information to better understanding of the real chemical nature of biological systems.

Since the pioneering work of Fenn and coworkers, [2] electrospray ionization mass spectrometry (ESI-MS) has been widely used and proven to be one of the most important analytical tools in chemistry, biochemistry, pharmacy and related sciences. ESI can gently transfer large biological molecules into gas-phase in multiply charged states even for an intact virus [3]. Usually, ESI employs a continuous-flow capillary for sampling, which means the procedures of dissolution, extraction and purification of the sample should be conducted before analysis. Therefore conventional ESI is seldom applied for direct analysis of biological tissues. However, it was later recognized that the electrosprayed plume and the charged droplets could interact with neutral molecules for secondary ionization. Thus some ESI-based ionization/ desorption methods, which can be used for direct analysis have been invented in recent years. Desorption electrospray ionization (DESI) [4] is one prominent example. In DESI, high-speed electrically charged drop- 
lets directly sprayed onto the sample surface and secondary ions would be generated during the process of surface collisions [5-7]. A similar technique related to DESI, where the solution was sprayed with very highspeed nebulization auxiliary gas but no voltage applied, is called desorption sonic spray ionization (DeSSI) [8] or easy ambient sonic-spray ionization (EASI) [9]. There are several other two-step techniques of coupling laser desorption and ESI. Shiea et al. have developed the electrosprayassisted laser desorption ionization (ELDI) which combines the properties of both ESI and laser desorption (LD) [10]. In ELDI, analytes are first desorbed by LD from the sample surfaces and then ionized or reacted with charged droplet in the ESI plume for secondary ionization. Techniques similar to ELDI include the addition of an organic acid matrix to enhance the sensitivity and extend the limit of molecular weight range of ELDI (MALDESI) [11], and the use of IR laser for the water-rich sample (LAESI) [12].

Recently, a modified ESI source, probe electrospray ionization (PESI), has also been developed in our laboratory [13]. A solid stainless needle is adopted to repeatedly load a small amount of sample solution by stitching the needle tip into the surface of biological tissues. Then, electrospray will be generated when a high voltage (e.g., $2.5 \mathrm{kV}$ ) is applied to the needle. No clogging problems but high tolerance of salts can be found with PESI [14].

This study continues our work on the direct analysis of biological samples by means of PESI-MS [13-15]. Previous works have demonstrated that PESI source is suitable for direct analysis of many kinds of samples. Herein, we report the application of PESI-MS for direct and in vivo analysis of tulip tissues. The aim of this study is to characterize phytochemicals in different regions of both tulip bulbs and plants, and to monitor the change of carbohydrate contents in tulip bulb scales during the first week of growth after dormancy release. The obtained data show that PESI-MS allows rapid and reliable characterization of chemical constituents in tulip bulbs.

\section{Experimental}

The side and front views of the experimental configuration of PESI setup were shown in Figure 1a and b, respectively. Stainless steel disposable acupuncture needles were purchased from Seirin (Shizuoka, Japan). These needles share the same diameter of about $140 \mu \mathrm{m}$ and a less-than-1 $\mu \mathrm{m}$ diameter tip [15]. An acupuncture needle was vertically aligned in the orthogonal direction to the axis of the ion sampling orifice. A linear actuator system was used to drive the needle up and down along the vertical axis. When the needle was driven up to the highest position, the vertical and horizontal distances between the tip of the needle and the apex of the ion sampling orifice were 2 and $3 \mathrm{~mm}$, respectively. The moving frequency of the needle was 3 $\mathrm{Hz}$ with an $8 \mathrm{~mm}$ moving stroke. The biological tissue sample was fixed on an XYZ sampling stage below the ion sampling orifice. The $X Y Z$ stage was adjusted manually to a position where the sample could just touch the tip of the needle when the needle was driven to the lowest position. A typical penetration depth for sampling was achieved by controlling the sample stage moving the sample up for about $0.5 \mathrm{~mm}$. A small amount of biofluid from the tissue sample could be loaded on the tip of the needle and electrosprayed when a high voltage of $2.5-3.0 \mathrm{kV}$ was applied to the needle [15]. The strongest ion signals could be observed when the needle reached the highest position [15]. Periodic sample loading and electrospraying processes were achieved by a linear actuator system.

MS experiments were carried out on an orthogonal time of flight (TOF) mass spectrometer (AccuTOF; JEOL, Akishima, Japan). Typical parameters for MS were: ion sampling orifice temperature, $80^{\circ} \mathrm{C}$; lens voltage, $10 \mathrm{~V}$; orifice 1 voltage, $200 \mathrm{~V}$; and orifice 2 voltage, $5 \mathrm{~V}$. All data were acquired and recorded under positive ionization mode using the analogue to digital converter (ADC)/ continuous averager ion detection system.

As an addition to our previously described PESI configurations [13-15], an auxiliary vapor generator was applied in current MS experiments. A commercial ESI emitter (JEOL, Akishima, Japan) was modified by attaching an automatic temperature controlled heater to the capillary of the emitter. When pure water was pumped through the capillary of the emitter continuously by a Harvard syringe pump (Pump 11; Harvard Apparatus, Holliston, MA, USA) at a flow-rate of 3

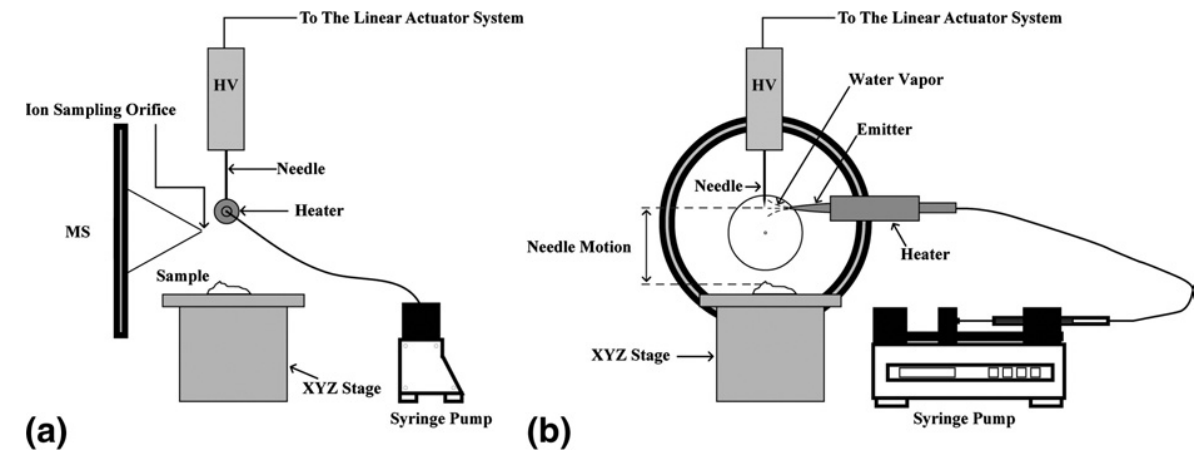

Figure 1. Schematic side view (a) and front view (b) of the PESI configuration. 
$\mu \mathrm{L} / \mathrm{min}$, water vapor was generated at the tip of the capillary of the emitter. This auxiliary vapor generator was fixed perpendicularly to the needle at the same height as the highest position of the needle motion. The distance between the emitter tip and the needle tip was about $10 \mathrm{~mm}$. When the temperature of the heater was set at $120^{\circ} \mathrm{C}$, generated water vapor was found to condense on the needle tip directly. No voltage was applied to this auxiliary vapor generator.

All regents used were of analytical grade or higher and used without further purification. Water was purified by a Milli-Q system (Millipore, Bedford, MA, USA). Tulip (Tulipa gesneriana L.) bulbs stored at $5{ }^{\circ} \mathrm{C}$ were provided by a local grower.

\section{Results and Discussions}

\section{Direct Analysis of Different Parts of a Tulip Bulb by PESI-MS}

Tulips are species of perennials from bulbs. During the dormancy period, a tulip bulb consists of 4 to 5 concentrically layered scales (fleshly leaves), a basal plate, and a central shoot. The section of a tulip bulb stored at $5{ }^{\circ} \mathrm{C}$ for about 20 weeks is shown in Figure 2a. The bulb stores carbohydrates including starch and fructan in the scales, which are energy and source of carbon for plants growth and flowering after dormancy. Starch and fructan can be enzymatically degraded to lower-molecularweight carbohydrates that are water-soluble and can be transferred to the shoot through the basal plate. The shoot contains several foliage leaves and a tender flower bud in the center. The basal plate is comprised of a complicated network of vascular bundles connecting the shoot, scales, and roots.

Since PESI is based on electrospray, it can only be applicable to liquid samples. In some cases, however, the surface of biological samples was not juicy enough; only a small amount of biofluid could be attached on the needle tip. During the upward motion of the needle, the attached biofluid might get dry, resulting in reduced and unstable ion signals. In addition, it was less likely that the entire liquid sample trapped on the needle was electrosprayed but some residues might be left on the surface of needle tip. This might cause cross-contamination due to the carryover of the previous sample loading. To avoid this kind of memory effect, an auxiliary water vapor generator was applied in this work. Herein, with the auxiliary water vapor generator's help, when the needle was at the highest position, water vapor would condense on the surface of the needle tip and then the dried compounds attached on the needle tip could be dissolved and electrosprayed. By using the auxiliary vapor generator, it was found that the stability and reproducibility were greatly improved compared with those without using it.

Figure $2 \mathrm{~b}-\mathrm{f}$ show the PESI mass spectra of direct analysis of a tulip bulb at different regions. Identifications of detected ions were based on the measured molecular weight information and references [16-18]. A series of ions corresponding to potassiated oligosaccharides at $m / z 381,543,705$, etc. separated by 162 Da were detected in almost every part of the tulip bulb with different relative intensities. Protonated arginine at $\mathrm{m} / \mathrm{z}$ 175 was also detected in the bulb with high intensities in scales and in the developing bud. Some previous studies [19-20] have reported that amino acids are ubiquitous in both bulbs and whole plants with different contents and play an important role on the nitrogenous metabolism. Arginine is the main free amino acid in tulip scales but only few in green leaves [19]. As shown in Figure $2 b$, e, and $f$, the foliage leaves contained much less arginine compared with the scale, in agreement with the work done by Zacharius et al. [19] For the developing bud (Figure 2c), protonated arginine at $m / z 175$ was detected as the main peak. Only lowmolecular-weight carbohydrates ions at $\mathrm{m} / \mathrm{z} 381$ and 543 were observed, which indicated that the developing bud contained less sugar-storing cells compared with other regions of tulip bulbs. Another ion at $m / z 579$ was also detected in this part. According to the reference [16], this ion could be identified as pelargonidin 3-rutinoside, an anthocyanin compound commonly detected in tulip petals. Anthocyanins are the flavonoid pigments that cause flowers to display various colors, and important to many diverse functions in plants [21]. It seemed that though the bud was developing, important compounds for flowering have already been synthesized and stored. Figure 2d shows the PESI spectrum of the basal plate part. Potassiated tuliposide A, the precursor compound of antifungal tulipalin A [22], was detected at $\mathrm{m} / \mathrm{z} 317$ as the main peak, and some carbohydrates were also detected with low intensity. Because the basal plate was the place of water and sugar transportation, the high concentration of tuliposide A indicated that tuliposide A might be biosynthesized in some specific region and transferred to other parts of the bulb through the basal plate. The vapor generated from the auxiliary vapor generator was pure water vapor, therefore only compounds with high water solubility such as carbohydrates and amino acids could be preferentially dissolved, loaded, and then electrosprayed. Some water-insoluble compounds like membrane lipids might be largely suppressed, which could be detected by other methods in tulip bulbs [23].

For the reason that the first scale (counted from the outermost) was often mechanically damaged [24] or infected by fungi [25], the second outermost scale was used in this study. The PESI-MS spectra of the inner and outer regions of the second outermost scale are shown in Figure 2e and f, respectively. It could be clearly seen that the inner region contained more disaccharides than the outer region. According to the reported microscopic observations of tulip bulbs, the inner and outer regions of tulip scale are functionally different [26]. The outer region is comprised of more sugar-storing cells but the inner region contains more cortex parenchyma cells. When treated in low temperature, polysaccharides 

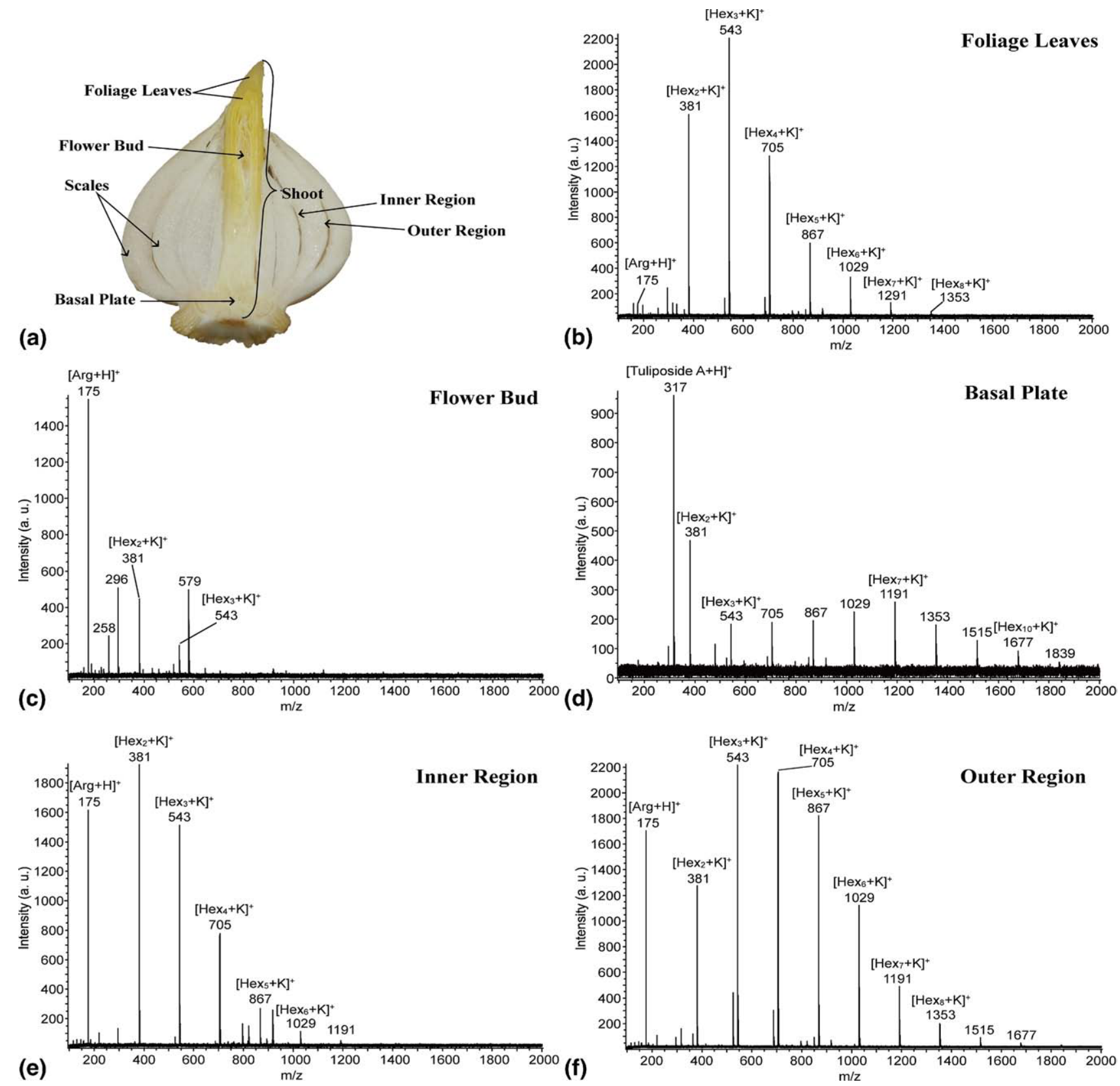

Figure 2. (a) section of a $5^{\circ} \mathrm{C}$ stored tulip bulb and PESI mass spectra of (b) the foliage leaves part, (c) the flower bud part, (d) the basal plate part, (e) the inner region of the second outermost scale, and

(f) the outer region of the second outermost scale, respectively.

granules could be enzymatically degraded to lowermolecular-weight carbohydrates. The degraded products would be transferred and accumulated in the inner cortex parenchyma cells and are sources of energy and carbon for the growth of the aerial part of the plant [27].

\section{Direct Analysis of Tulip Petals and Leaves by PESI-MS}

To compare the difference of phytochemicals in tulip bulbs and tulip plants, several tulip bulbs were planted in pots at room temperature. After 6 weeks, they started to bloom. Fresh petals and leaves were collected and used for analysis without any further preparation. Figure $3 a$ and $b$ show PESI mass spectra of the red part and the yellow part of a tulip petal. Potassiated tuliposide A, tuliposide $\mathrm{B}$, and disaccharide $\left(\mathrm{Hex}_{2}\right)$ at $m / z 317,333$, and 381 were detected in both spectra, respectively. Only from the red part, some pelargonidin type pigments were detected, including pelargonidin, pelargonidin 3-rutinoside, and pelargonidin 3-(2'"'-acetylrutinoside) at $m / z 271,579$, and 621, respectively. Figure $3 a$ also indicates that these pelargonidin type anthocyanins might contribute greatly to the bright red color of tulip flowers. 

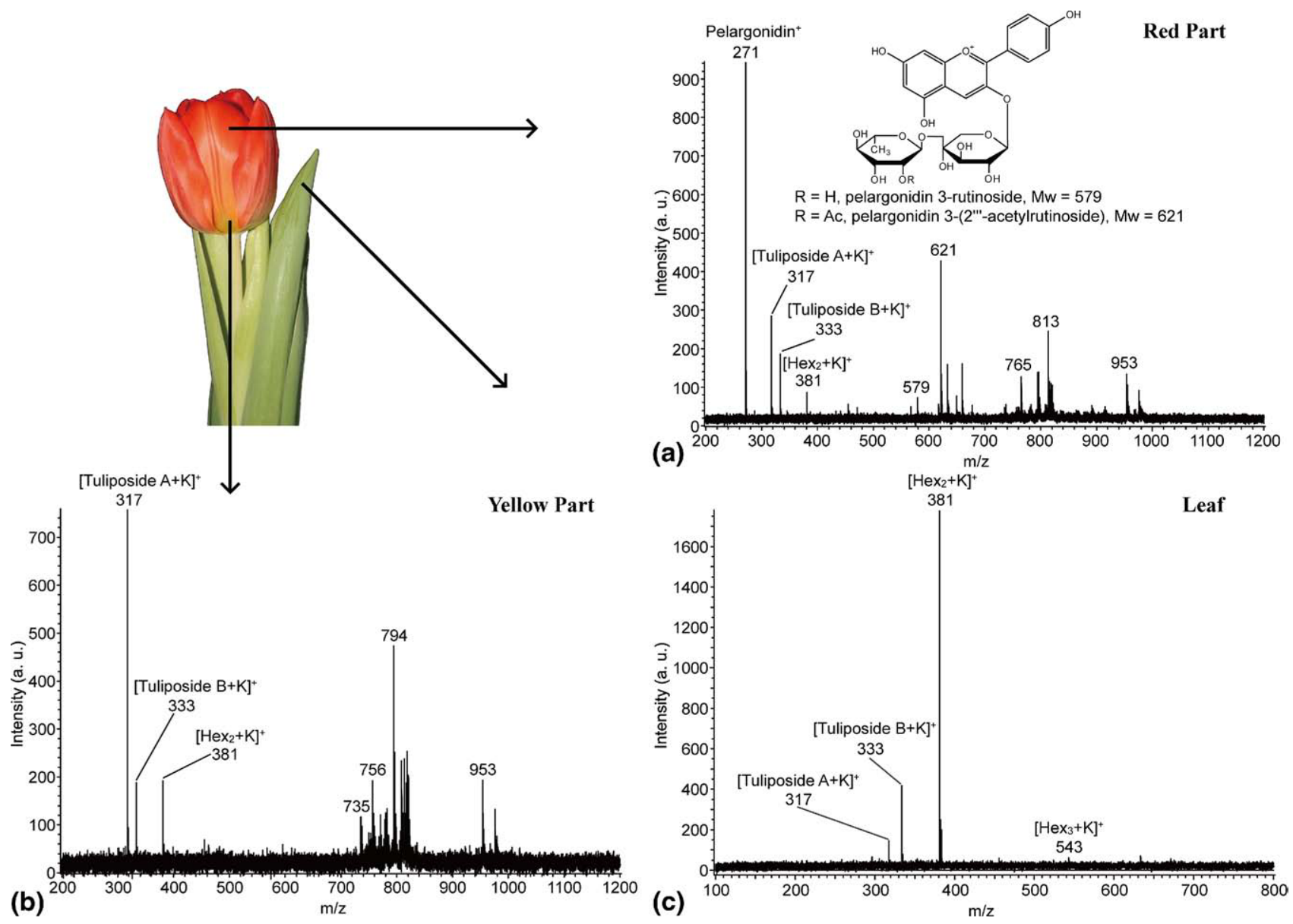

Figure 3. PESI mass spectra of the aerial part of a tulip plant, (a) the red part of the petal, (b) the yellow part of the petal, and (c) leaf, respectively.

Figure 3c shows the PESI mass spectrum of the leave of a tulip plant. Only four peaks at $m / z 317,333,381$, and 543 , corresponding to potassiated tuliposide A, tuliposide $\mathrm{B}$, disaccharide $\left(\mathrm{Hex}_{2}\right)$, and trisaccharide $\left(\mathrm{Hex}_{3}\right)$ were detected, respectively. Compared with the spectrum of foliage leaves of bulbs shown in Figure 2c, it could be clearly seen that carbohydrates stored in foliage leaves in tulip bulbs would be degraded and used during the growth process. Both petals and leaves contain a certain content of tuliposide A and B, which can be regarded as bioactive compounds with self-protective properties. But in tulip bulbs, these compounds could not be detected in both foliage leaves and flower buds parts, which indicated that these compounds might be marker compounds of growth of tulips.

\section{Every Other Day In Vivo Monitoring of the Change of Carbohydrate Content in Tulip Bulbs by PESI-MS}

Carbohydrate metabolism is an important part of total metabolism in plants. Revealing the time-course of changes of carbohydrate content can contribute to a better understanding of carbohydrate metabolism in plants. Furthermore, these changes may be used as physiological markers, indicating the timing of developmental transitions in plants [28]. Direct analysis methods for monitoring changes of carbohydrate content will be favorable because time-consuming and complicated sample preparation procedures can be avoided. Because of advantages such as low-sample consumption, direct sampling, and high tolerance to salts [14], PESI-MS could be one ideal technique for direct monitoring of chemical changes in biological systems in vivo.

PESI needs to stitch the sample for sampling. Although the sample consumption of PESI was small, the needle would still damage cells on the top surface. The stitched region was found to become yellow or slightly brown at $5 \mathrm{~mm}$ or less in diameter. In tulip bulbs, there exist antifungal compounds (tulipalines) and their precursors (tuliposides). If one part of tulip tissues gets wounded, tulipalines will be released, not only from the wounded part but from adjacent cells [25]. Observing the occurrence of brown spots in tulip bulb scales induced by fungal infections, Bergman [29] suggested that the rapid release of tulipalines from cells adjacent to the infected place in the tulip bulb scales would be 
the reason of cell death in this area by the reaction of tulipalines with other cell components, and further infections would be effectively inhibited by the encapsulation of the infection area by a layer of dead cells. Not all parts of bulb scales but only a limited area of several millimeters in diameter will be affected to release tulipalines according to literatures $[25,29]$.

To demonstrate how PESI-MS could be applied to in vivo monitoring of changes of carbohydrate content in tulip bulbs, we assumed that both the changes of chemicals and reactions of tulipalines with other chemicals only happen in the yellow region, not in the whole bulb. So a different region in the bulb scale was used for every measurement to exclude interferences brought on by previous measurements.

To investigate changes of carbohydrate content of tulip bulb scales during the initial growth period, in vivo experiments were conducted to four tulip bulbs that were stored at $5{ }^{\circ} \mathrm{C}$ for about 20 weeks. At the beginning, the tunics (the outer covering of the bulb) and the first outermost scales of all four tulip bulbs were carefully excised. Then the bulbs were put on a piece of mesh gauze, covered, on a water container. It was made possible that only roots of these bulbs were soaked in water when the water container was filled with enough water. Only purified water was used to exclude the role of minerals in the water. This container and four bulbs were kept under room temperature for 1 week. PESI-MS measurements were conducted directly to the outermost (originally the second outermost) scales of the bulbs. No excising or cutting for sampling was made to these tulip bulbs. PESI-MS data were collected a total of four times only, from day 0 , the starting day when the 20 -week storage at $5{ }^{\circ} \mathrm{C}$ of these bulbs was completed, to day 6, every other day. As tulip bulbs started to grow, shoots became much larger and longer, which made direct sampling to these bulbs more and more difficult due to the limitation of the configuration of PESI-MS. Considering some parts except the sampling location of these tulip bulbs would get wounded during the sampling process if the shoots became too long, the period of in vivo monitoring of the change of carbohydrate contents was set to the first week after dormancy release.

Figure 4 shows photographs of four tulip bulbs taken before every-other-day measurement during the first week of growth. It could be seen that the shoot of every bulb had elongated a little and the tips had become red in the first 2 days. In the following 4 days, the shoot developed much faster and turned from yellow to green. Conversely, the root grew very fast at the beginning but slowly in the following 4 days. Figure 5 shows the every-other-day PESI-MS measurements of the no. 3 bulb (counted from the left side in Figure 4). It could be clearly seen that the carbohydrate content in scales had a decreasing tendency during the first week of growth, while obvious morphologic changes happened to the bulbs. Other bulbs had the same tendency of changes of carbohydrate content as the no. 3 bulb (data not shown). These results agree with those published previously by using other analytical methods [17, 18, 26, 30-31].

The rapid growth of tulip bulbs may be ascribed to cell expansion [32]. Usually, cell expansion needs readily available hexoses for increased metabolism and biosynthesis of new materials. It is well known that starch is the major storage of carbohydrate in tulip bulbs and $\alpha$-amylase is the key enzyme for initiation of starch degradation in most tissues [30]. Our present
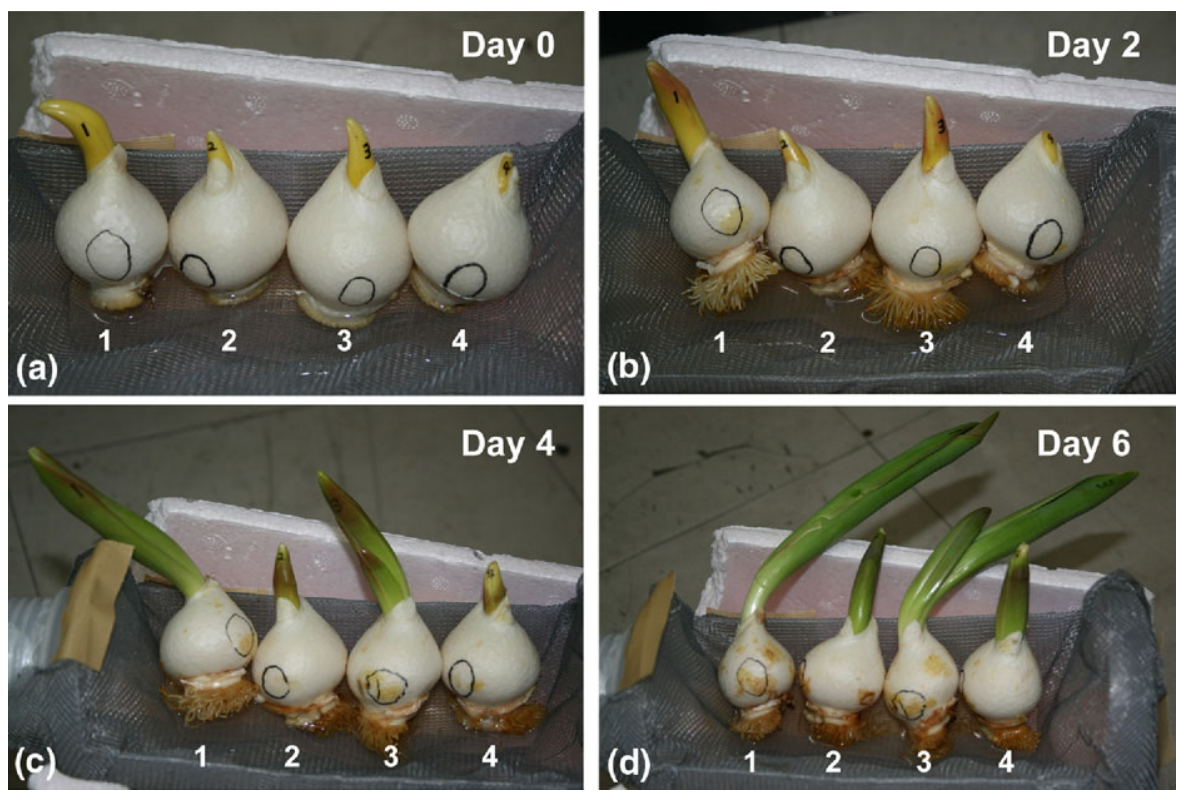

Figure 4. From (a)-(d): Photographs of four tulip bulbs during the first week of growth taken at the day $0,2,4$, and 6 respectively. These bulbs were numbered from left to right, from 1 to 4 . All pictures were taken before PESI-MS measurements. 

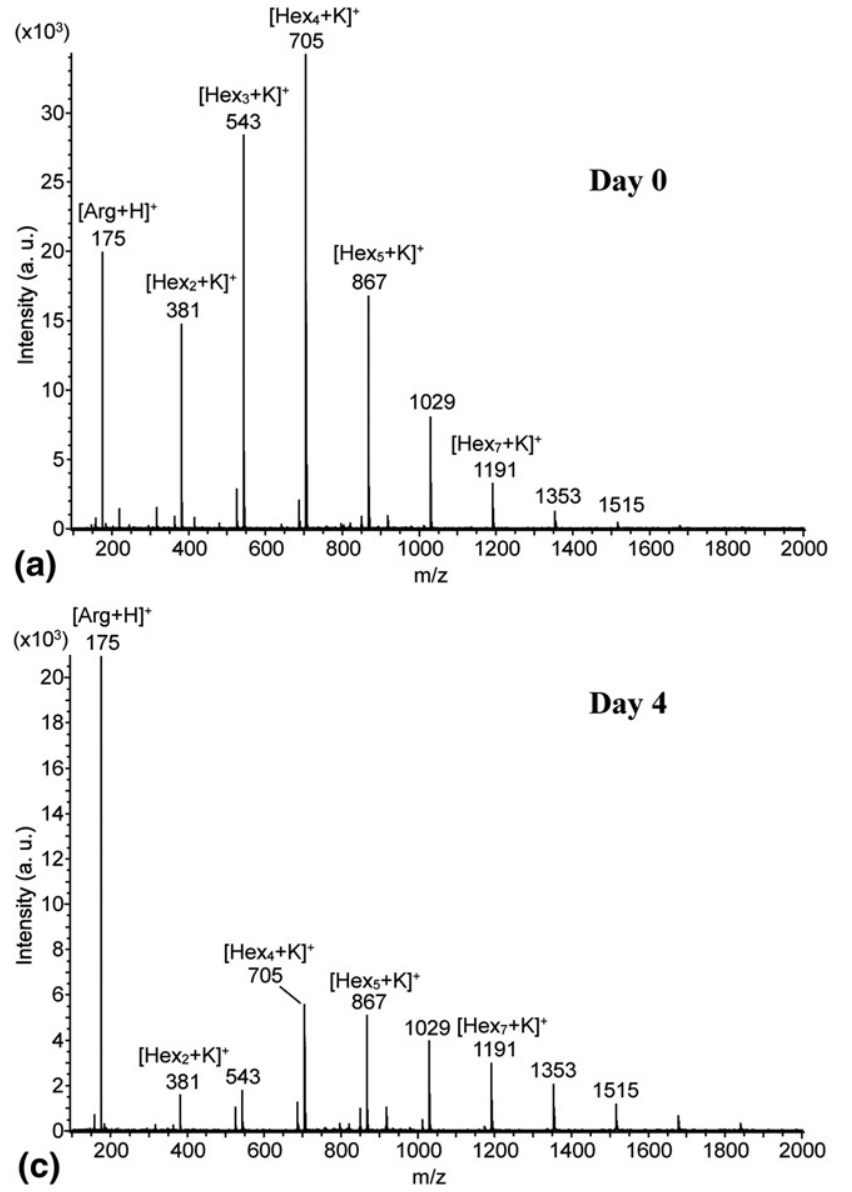
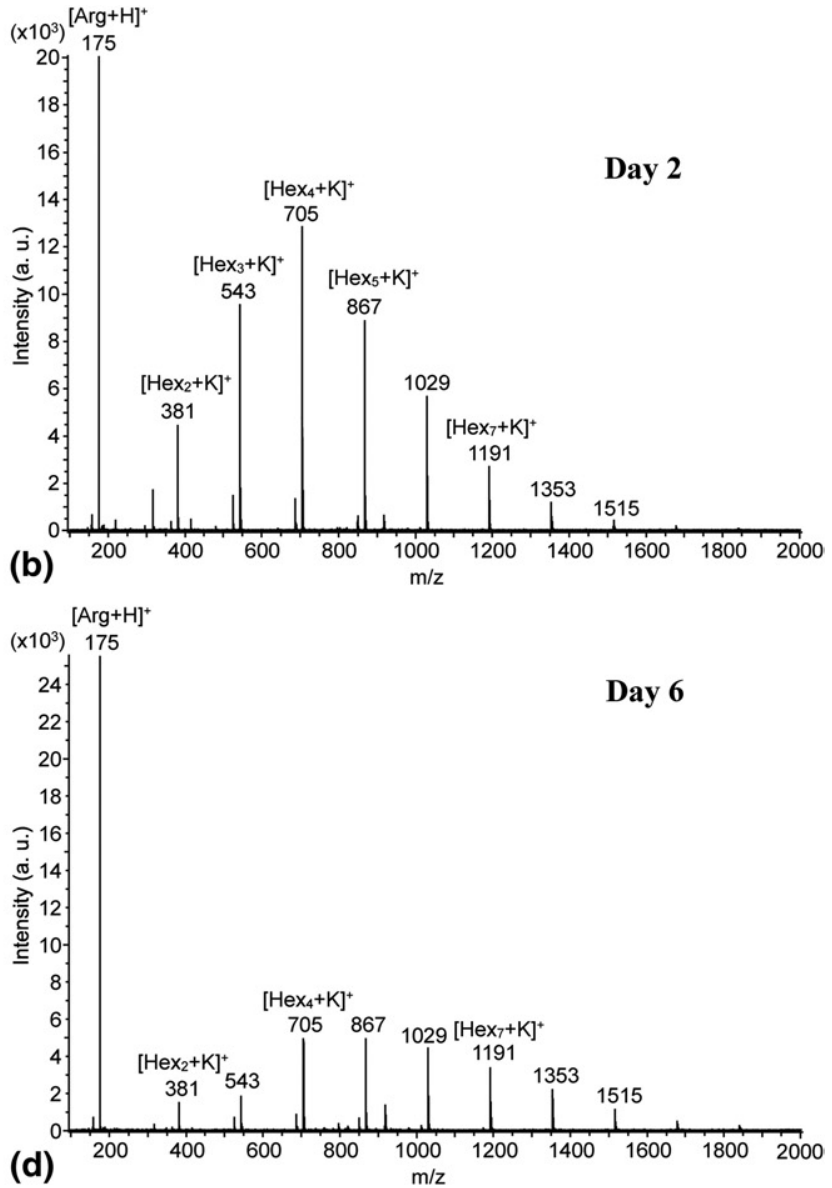

Figure 5. From (a)-(d): PESI mass spectra of the No. 3 bulb (numbered in Figure 4) during the first week of growth measured at the day $0,2,4$ and 6 respectively.

results also indicate that the activity of $\alpha$-amylase may increase in the first week of growth of tulip bulbs. The increased $\alpha$-amylase activity may be the instinctive response to changes in temperature and contact with water.

\section{Conclusions}

In this study, we have demonstrated the application of PESI-MS for direct analysis of tulip tissues, including bulbs and plants. Strong ion signals could be observed in every part of tulip tissues. Our results have shown that though tulip bulbs were in dormancy, some important compounds have already been biosynthesized and stored in some specific regions. Applying PESI-MS for in vivo analysis of carbohydrate changes in tulip bulb scales has also provided direct evidence for changes of carbohydrate content of tulip bulbs in the initial growth period.

The current research highlights the application of PESI-MS for direct analysis of plant tissues and could be extended to other biological samples. Results of this study have also suggested that PESI can be applied to monitoring/observing of changes of chemical content and discovery of new chemical constituents in biologi- cal samples. This method is simple, fast, and reproducible. Further research will be focused on applying PESI as a monitoring tool for metabolism changes and changes of chemical content in some plants or microbes, which may be the reflection of environmental changes.

\section{Acknowledgments}

The authors acknowledge support for this work by the Grants-inAid for Scientific Research (S) and Development of System and Technology for Advanced Measurement and Analysis Program (SENTAN) from Japan Science and Technology Agency (JST). L.C.C. acknowledges the financial support from the Japan Society for the Promotion of Science (JSPS).

\section{References}

1. Cowan, M. M. Plant Products as Antimicrobial Agents. Clin. Microbiol. Rev. 1999, 12, 564-582.

2. Fenn, J. B.; Mann, M.; Meng, C. K.; Wong, S. F.; Whitehouse, C. M. Electrospray Ionization for Mass Spectrometry of Large Biomolecules. Science 1989, 246, 64-71.

3. Fuerstenau, S. D.; Benner, W. H.; Thomas, J. J.; Brugidou, C.; Bothner, B. Siuzdak, G. Mass Spectrometry of an Intact Virus. Angew. Chem. Int. Ed. 2001, 40, 541-544.

4. Takats, Z.; Wiseman, J. M.; Gologan, B.; Cooks, R. G. Mass Spectrometry Sampling Under Ambient Conditions with Desorption Electrospray Ionization. Science 2004, 306, 471-473. 
5. Volny, M.; Venter, A.; Smith, S. A.; Pazzi, M.; Cooks, R. G. Surface Effects and Electrochemical Cell Capacitance in Desorption Electrospray Ionization. Analyst 2008, 133, 525-531.

6. Cooks, R. G.; Ouyang, Z.; Takats, Z.; Wiseman, J. M. Ambient Mass Spectrometry. Science 2006, 311, 1566-1570.

7. Ifa, D. R.; Wiseman, J. M.; Song, Q.; Cooks, R. G. Development of Capabilities for Imaging Mass Spectrometry Under Ambient Conditions with Desorption Electrospray Ionization (DESI). Int. J. Mass Spectrom. 2007, 259, 8-15.

8. Haddad, R.; Sparrapan, R.; Eberlin, M. N. Desorption Sonic Spray Ionization for (High) Voltage-Free Ambient Mass Spectrometry. Rapid Commun. Mass Spectrom. 2006, 20, 2901-2905.

9. Haddad, R.; Sparrapan, R.; Kotiaho, T.; Eberlin, M. N. Easy Ambient Sonic-Spray Ionization-Membrane Interface Mass Spectrometry for Direct Analysis of Solution Constituents. Anal. Chem. 2008, 80, 898-903.

10. Huang, M.Z.; Hsu, J.; Wu, C.I.; Lin, S.Y.; Ma, Y.L.; Cheng, T.L.; Shiea, J. Characterization of the Chemical Components on the Surface of Different Solids with Electrospray-Assisted Laser Desorption Ionization Mass Spectrometry. Rapid Commun. Mass Spectrom. 2007, 21, 1767-1775.

11. Sampson, J. S.; Hawkridge, A. M.; Muddiman, D. C. Generation and Detection of Multiply-Charged Peptides and Proteins by Matrix-Assisted Laser Desorption Electrospray Ionization (MALDESI) Fourier Transform Ion Cyclotron Resonance Mass Spectrometry. J. Am. Soc. Mass Spectrom. 2006, 17, 1712-1716.

12. Nemes, P.; Vertes, A. Laser Ablation Electrospray Ionization for Atmospheric Pressure, In Vivo, and Imaging Mass Spectrometry. Anal. Chem. 2007, 79, 8098-8106.

13. Hiraoka, K.; Nishidate, K.; Mori, K.; Asakawa, D.; Suzuki, S. Development of Probe Electrospray Using a Solid Needle. Rapid Commun. Mass Spectrom. 2007, 21, 3139-3144.

14. Chen, L. C.; Nishidate, K.; Saito, Y.; Mori, K.; Asakawa, D.; Takeda, S.; Kubota, T.; Terada, N.; Hashimoto, Y.; Hori, H.; Hiraoka, K. Application of Probe Electrospray to Direct Ambient Analysis of Biological Samples. Rapid Commun. Mass Spectrom. 2008, 22, 2366-2374.

15. Chen, L. C.; Nishidate, K.; Saito, Y.; Mori, K.; Asakawa, D.; Takeda, S.; Kubota, T.; Hori, H.; Hiraoka, K. Characteristics of Probe Electrospray Generated from a Solid Needle. J. Phys. Chem. B 2008, 112, 11164-11170.

16. Torskangerpoll, K.; Nørbæk, R.; Nodland, E.; Øvstedal, D. O.; Andersen,. Ø. M. Anthocyanin content of Tulipa Species and Cultivars and Its Impact on Tepal Colours. Biochem. Syst. Ecol. 2005, 33, 499-510.

17. Gholipour, Y.; Nonami, H.; Erra-Balsells, R. In Situ Analysis of Plant Tissue Underivatized Carbohydrates and On-Probe Enzymatic Degraded Starch by Matrix-Assisted Laser Desorption/Ionization Timeof-Flight Mass Spectrometry by Using Carbon Nanotubes as Matrix. Anal. Biochem. 2008, 383, 159-167.

18. Gholipour, Y.; Nonami, H.; Erra-Balsells, R. Application of Pressure Probe and UV-MALDI-TOF MS for Direct Analysis of Plant Underivat- ized Carbohydrates in Subpicoliter Single-Cell Cytoplasm Extract. J. Am. Soc. Mass Spectrom. 2008, 19, 1841-1848.

19. Zacharius, R. M.; Cathey, H. M.; Steward, F. C. Nitrogenous Compounds and Nitrogen Metabolism in the Liliaceae: III. Changes in the Soluble Nitrogen Compounds of the Tulip and Their Relation to Flower Formation in the Bulb. Ann. Bot. 1957, 21, 193-201.

20. McKee, H. S. Nitrogen Metabolism in Plants; Clarendon Press: Oxford, 1962; p. 417.

21. Holton, T. A.; Cornish, E. C. Genetics and Biochemistry of Anthocyanin Biosynthesis. Plant Cell 1995, 7, 1071-1083.

22. van Baarlen, P.; Legendre, L.; van Kan, J. Plant Defence Compounds Against Botrytis Infection. In: Botrytis: Biology, Pathology and Control, Elad, Y.; Williamson, B.; Tudzynski, P.; Delen, N., Eds.; Kluwer Academic Press: Dordrecht, 2004; pp 143-161.

23. Walch, K.; van Hasselt, P. R. The Influence of Low Temperature on the Membrane Lipid Composition and Flowering Capacity of Tulip Bulbs. Acta Hort. 1991, 298, 345-354.

24. van Kilsdonk, M. G.; Nicolay, K.; Franssen, J. M.; Kolloffel, C. Bud Abortion in Tulip Bulbs Studied by Magnetic Resonance Imaging. J. Exp. Bot. 2002, 53, 1603-1611.

25. van Rossum, M. W. P. C.; Alberda, M.; van der Plas, L. H. W. Tulipaline and Tuliposide in Cultured Explants of Tulip Bulb Scales. Phytochemistry 1998, 49, 723-729.

26. Kamenetsky, R.; Zemah, H.; Ranwala, A. P.; Vergeldt, F.; Ranwala, N. K.; Miller, W. B.; Van As, H.; Bendel, P. Water Status and Carbohydrate Pools in Tulip Bulbs During Dormancy Release. New Phytol. 2003, 158, 109-118.

27. Iwaya-Inoue, M.; Motooka, K.; Ishida, N.; Koizumi, M.; Kano, H. Chilling Effects for Normal Growth of Tulip Bulbs Estimated by MRI. Acta Hort. 1996, 440, 407-412.

28. Naor, V.; Kigel, J.; Ben-Tal, Y.; Ziv, M. Variation in Endogenous Gibberellins, Abscissic Acid, and Carbohydrate Content During the Growth Cycle of Colored Zantedeschia spp., a Tuberous Geophyte. J. Plant Growth Regul. 2008, 27, 211-220.

29. Bergman, B. Presence of a Substance in the White Skin of Young Tulip Bulbs That Inhibits Growth of Fusarium oxysporum. Eur. J. Plant Pathol. 1966, 72, 222-230.

30. Geng, X.; Sato, A.; Okubo, H.; Saniewski, M. Changes in Carbohydrate and ABA Content in Tulip Bulbs During Storage. J. Fac. Agr. Kyushu Univ. 2007, 52, 315-319.

31. Ranwala, A.; Miller, W. Gibberellin-Mediated Changes in Carbohydrate Metabolism During Flower Sstalk Elongation in Tulips. Plant Growth Regul. 2008, 55, 241-248.

32. Gilford, J.; Rees, A. Growth of the Tulip Shoot. Sci. Hort. 1973, 1, 143-156. 